\title{
LOCALIZATION OF SPECTRIN IN MAMMALIAN BRAIN ${ }^{1}$
}

\author{
IAN S. ZAGON, ${ }^{*, 2}$ PATRICIA J. MCLAUGHLIN, ${ }^{*}$ AND STEVEN R. GOODMAN \\ Departments of *Anatomy and $\ddagger$ Physiology, The Milton S. Hershey Medical Center, The Pennsylvania State University, \\ Hershey, Pennsylvania 17033
}

Received February 14, 1984; Revised May 7, 1984; Accepted May 16, 1984

\begin{abstract}
Spectrin is a major skeletal component of the erythrocyte membrane and is essential in controlling cell shape and structural stability. The brain has also been found to be rich in an immunoreactive and structural analogue of spectrin. In the present study, spectrin was localized in the mouse brain by indirect immunofluorescence using an antibody to erythrocyte spectrin that cross-reacts specifically with the $\alpha$ and $\beta$ subunits of brain spectrin. Spectrin antigens were concentrated in neuronal perikarya and cell processes. Synaptic structures and axons were observed to have little detectable spectrin antigen by immunofluorescence methodology. The cell bodies of glia had a less intense immunoreactivity in contrast to neurons, and glial processes and myelin were unstained. Cell nuclei of neural cells were not fluorescent. These results show that $(a)$ spectrin is found in all regions of mammalian brain and its intensity corresponds to neural cell density, $(b)$ different neural cell types contain variable spectrin content, and $(c)$ within a single neural cell, the regional disposition of spectrin varies.
\end{abstract}

Spectrin is a large fibrous protein which, in association with actin protofilaments and several other proteins, forms the erythrocyte membrane skeleton. This membrane skeleton is essential for the maintenance of erythrocyte shape, reversible deformability, and membrane structural integrity, in addition to controlling the lateral mobility of integral membrane proteins (for review, see Goodman and Shiffer, 1983). In the erythrocyte, this membrane skeleton is associated with the bilayer core through interaction of the spectrin $(\alpha \beta)_{2}$ tetramer $(\alpha=240,000$ daltons and $\beta=220,000$ daltons; also termed bands 1 and 2) (Steck, 1974) with a peripheral membrane protein termed syndein ( $\mathrm{Yu}$ and Goodman, 1979) or ankyrin (Bennett and Stenbuck, 1979), which is in turn associated with the integral membrane glycoprotein band 3 (Bennett and Stenbuck, 1980). Protein 4.1, an 80,000-dalton peripheral membrane protein, converts a low affinity spectrin-actin interaction into a high affinity spectrin-protein 4.1-actin ternary complex, thereby forming the two-dimensional skeletal meshwork (for review, see Goodman and Shiffer, 1983), as well as serving as a secondary site for membrane attachment (Shiffer and Goodman, 1983, 1984).

Our laboratory originally noted that molecules which are both immunologically and structurally related to erythrocyte spectrin exist in nonerythroid cells, and this family of related molecules was referred to as nonerythroid spectrin-like proteins (Goodman et al., 1981). Subsequent immunofluorescence studies from several laboratories have demonstrated the widespread

\footnotetext{
${ }^{1}$ This work was supported in part by National Institutes of Health Grant NS-21246 to I. S. Z. and Grants NS-19357 and HL-26059 to S. R. G. S. R. G. is an Established Investigator of the American Heart Association. We thank Dr. Carey Balaban for the helpful discussions during the course of this research.

${ }^{2}$ To whom correspondence should be addressed.
}

occurrence of nonerythroid spectrin-like molecules in the cortical cytoplasm of nearly every mammalian and avian cell or tissue studied (e.g., Levine and Willard, 1981; Burridge et al., 1982; Glenney et al., 1982a; Repasky et al., 1982; Glenney and Glenney, 1983a, b; Goodman and Shiffer, 1983 (review); Goodman et al., 1983, 1984a; Lazarides and Nelson, 1983a, b; Casoria et al., 1984).

The brain is rich in an immunoreactive and structural analogue of spectrin (Bennett et al., 1982; Burridge et al., 1982; Glenney et al., 1982b; Goodman et al., 1983, 1984a, b; Casoria et al., 1984) which had been studied as a stimulator of an actomyosin $\mathrm{Mg}^{2+}$-ATPase (termed brain actin-binding protein; Shimo-Oka and Watanbe, 1981), as an axonally transported protein found in the cortical cytoplasm of neurons as well as other cell types (termed fodrin; Levine and Willard, 1981), and as a calmodulin-binding protein (Kakiuchi et al., 1981; subsequently termed calspectin), before its immunological and structural relationship with erythrocyte spectrin was fully appreciated. As this protein fits well into the class of nonerythroid spectrin-like molecules, we will refer to it here as brain spectrin.

Mouse brain spectrin is a large, asymmetric, 972,000-dalton, $10.5 \mathrm{~S}(\alpha \beta)_{2}$ tetramer which constitutes $2.3 \%$ of crude brain membrane protein (Goodman et al., 1983, 1984a; Casoria et al., 1984). Mouse brain spectrin consists of two subunits: 240,000 daltons ( $\alpha$ subunit) and 235,000 daltons ( $\beta$ subunit). Proteins of similar structure and subunit composition have been isolated from chicken, pig, and cow brain membranes (Bennett et al., 1982; Burridge et al., 1982; Glenney et al., 1982a). The mouse brain 235,000 -dalton $\beta$ subunit and mouse red blood cell (RBC) 220,000 -dalton $\beta$ subunit of spectrin are both phophorylated by a membrane-associated cAMP-independent protein kinase (Goodman et al., 1984a). Rotary shadowed images of brain spectrin demonstrate a long, flexible rod of $\sim 2,000 \AA$ contour length, with the two strands woven into a tight double helix with few or no gaps (Bennett et al., 1982; Glenney et al., 1982a), 
a structure which is strikingly similar to that of erythrocyte spectrin (Shotton et al., 1979). Brain spectrin crosslinks Factin in solution (Levine and Willard,1981; Bennett et al., 1982; Burridge et al., 1982; Glenney et al., 1982a). Rotary shadowed images of the interaction demonstrate that brain spectrin (like erythrocyte spectrin tetramer) is bivalent, binding laterally to $\mathrm{F}$-actin in an end-on orientation (Glenney et al., 1982c). Other functional characteristics shared by brain and erythrocyte spectrin include binding sites for erythrocyte syndein/ankyrin (Bennett et al., 1982; Burridge et al., 1982), calmodulin (Carlin et al., 1982; Glenney et al., 1982b; Kakiuchi et al., 1982; Palfrey et al., 1982), and erythrocyte protein 4.1 (Burns et al., 1983). Although brain and erythrocyte spectrin share common antigenic sites, morphology, subunit composition, and functional sites, two-dimensional peptide mapping analysis of radioiodinated $\alpha$ and $\beta$ subunits indicate only limited homology between brain and erythrocyte spectrin subunits in the mouse (Goodman et al., 1984a) or pig (Bennett et al., 1982; Glenney and Glenney, 1983a, b). It has therefore been concluded that mammalian brain and erythrocyte spectrin are distinct gene products, which share common functional regions such as actin-, syndein-, protein 4.1-, and calmodulin-binding sites, separated by functionally silent domains of variable sequence (for review, see Goodman and Shiffer, 1983).

To understand the function of brain spectrin, in addition to in vitro studies describing its structure and binding capacities, we must have an accurate and complete understanding of the localization of this molecule within mammalian neural tissue. Although several laboratories, including our own, have presented individual pictures of selected neural tissue stained with antibodies against RBC or brain spectrin (Levine and Willard, 1981; Goodman et al., 1983, 1984a, b; Lazarides and Nelson, 1983a, b; Casoria et al., 1984), no detailed account of the localization of brain spectrin in mammalian brain has appeared. In this report we describe the results of a comprehensive examination in regard to the immunocytochemical localization of brain spectrin within mouse brain.

\section{Materials and Methods}

Animals. Adult male C57BL/6 mice (Jackson Laboratories, Bar Harbor, ME) were utilized in this study and were housed under controlled conditions (Zagon and McLaughlin, 1981). All animals were allowed at least 5 days to acclimate to their surroundings prior to the beginning of experimentation.

Spectrin isolation and the characterization of spectrin antibodies. Brain and erythrocyte membrane preparations in the presence of potent protease inhibitors were obtained as previously described (Goodman et al., 1983). Brain and erythrocyte spectrin were purified by low ionic strength extraction of isolated membranes followed by rate zonal sedimentation through sucrose gradients (Goodman et al., 1983). Native purified mouse RBC spectrin heterodimers were used as immunogens for preparation of antibodies in rabbits. Antibodies were characterized by immunoautoradiography utilizing a gel overlay method (Goodman et al., 1981).

SDS-PAGE was performed on 1.5-mm-thick slab gels using the continuous buffer system of Fairbanks et al. (1971) at $50 \mathrm{~mA} / \mathrm{slab}$, with the modifications previously described (Casoria et al., 1984; Goodman et al., 1984a). For certain experiments in which we wanted clear separation of the $240,000-$ and $235,000-M_{\mathrm{r}}$ subunits of mouse brain spectrin, electrophoresis was allowed to proceed $4 \mathrm{hr}$ beyond the time at which the tracking dye reached the bottom of the gel. Autoradiog. raphy was performed using Kodak X-OMAT XAR film with a DuPont Cronex Lightning Plus intensifying screen at $-20^{\circ} \mathrm{C}$ for the time given in the figure legends.

Indirect immunofluorescence. Mice were anesthetized with $0.3 \%$ chloral hydrate and perfused through the heart with phosphatebuffered saline (PBS) at an air pressure of $120 \mathrm{~mm} \mathrm{Hg}$; this technique was found to be very effective in minimizing the appearance of erythrocytes in our immunocytochemical preparations. Animals were immediately decapitated, the entire brain was rapidly removed, and the tissues were frozen in Freon-12. Sections of $10 \mu \mathrm{m}$ were obtained with a Slee cryostat at $-15^{\circ} \mathrm{C}$, mounted on clean coverglasses, air dried at $-15^{\circ} \mathrm{C}$ for at least $20 \mathrm{~min}$, fixed in ethanol $(95 \%)$ at $4^{\circ} \mathrm{C}$ for $30 \mathrm{~min}$, and made permeable to the antibody by immersion in absolute acetone at $4^{\circ} \mathrm{C}$ for $20 \mathrm{~min}$. Fixed tissues were stored at $-70^{\circ} \mathrm{C}$ before immunofluorescent staining.

Tissue sections were washed in $8 \mathrm{ml}$ of PBS $\left(10 \mathrm{mM} \mathrm{KPO}_{4}, 150 \mathrm{mM}\right.$ $\mathrm{NaCl}$ ), pH 7.5 , for $10 \mathrm{~min}$ at $20^{\circ} \mathrm{C}$. One hundred microliters of antispectrin IgG $(100 \mu \mathrm{g} / \mathrm{ml})$, or IgG preincubated with a 10 -fold excess of spectrin, or nonimmune IgG $(200 \mu \mathrm{g} / \mathrm{ml})$ in PBS and $2 \%$ goat serum (PBS-NGS) were layered onto each sample, and the tissues were incubated for $3 \mathrm{hr}$ at $20^{\circ} \mathrm{C}$ in a humidified chamber. Samples were washed three times with $8 \mathrm{ml}$ of PBS-NGS and $0.1 \%$ Triton X-100 for $30 \mathrm{~min} /$ wash at $20^{\circ} \mathrm{C}$, incubated with $8 \mathrm{ml}$ of rhodamine-conjugated goat anti-rabbit IgG (N. L. Cappell Laboratories) (1:150 dilution from stock) in PBS-NGS containing $0.1 \%$ Triton X-100 for $30 \mathrm{~min}$ at $37^{\circ} \mathrm{C}$, and washed four times with PBS-NGS $\left(8 \mathrm{ml}, 30 \mathrm{~min} / \mathrm{wash}, 20^{\circ} \mathrm{C}\right)$ and once in PBS $\left(8 \mathrm{ml}, 30 \mathrm{~min}, 20^{\circ} \mathrm{C}\right)$. Specimens were mounted on glass slides with buffered glycerol ( $60 \%$ glycerol, $\left.40 \% 0.4 \mathrm{M} \mathrm{KHCO}_{3}, \mathrm{pH} 8.6\right)$ and examined with a Zeiss Universal microscope equipped with epifluorescence, phase contrast, and brightfield optics.

The brains of at least two animals were sectioned either sagittally or coronally. A complete series of sections was examined, with five serial sections obtained every $100 \mu \mathrm{m}$. In addition to staining at least one scetion of every five sections with anti-RBC spectrin $\mathrm{IgG}$, one section was stained utilizing the gallocyanin method (Einarson, 1951) and used for general orientation and morphology, and another section was stained with Zagon's modification of the Protargol technique (Zagon and Lasher, 1977; Zagon and Haring, 1982) for identification of neurons and nerve fibers.

In some cases, following visualization and photography of fluorescence material, sections were reprocessed for Protargol or gallocyanin staining for further examination. This procedure was a powerful tool in precisely identifying which neural elements were fluorescence positive or negative.

\section{Results}

\section{Characterization of the anti-mouse $R B C$ spectrin $I g G$}

In previous studies (Casoria et al., 1984; Goodman et al., 1984a) we have demonstrated that our antibody raised in rabbits against purified native mouse RBC spectrin heterodimer is specific for spectrin, staining only the $\alpha$ and $\beta$ subunits in immunoautoradiographic characterization of total mouse erythrocyte membrane protein. In Figure 1, we show that this monospecific anti-RBC spectrin IgG stains only brain spectrin when demyelinated brain membrane protein is separated by SDS-PAGE, stained with the antibody, and counterstained with ${ }^{125}$ I-Protein A. As the 240,000- and 235,000-dalton $\alpha$ and $\beta$ subunits of brain spectrin are not well resolved on standard SDS-PAGE, we have extended the electrophoresis time $4 \mathrm{hr}$ beyond the time that the tracking dye reached the bottom of the gel. This procedure allowed clear resolution of the $\alpha$ and $\beta$ subunits of brain spectrin (Fig. 2). When demyelinated brain membrane proteins or purified brain spectrin are separated by extending electrophoresis time and stained with anti-mouse $\mathrm{RBC}$ spectrin IgG, we found that the antibody cross-reacts specifically with both the $\alpha$ and $\beta$ subunits of brain spectrin, with preferential staining of the $\alpha$ subunit (Fig. 2). Because our antibody against mouse RBC spectrin cross-reacts only with the $\alpha$ and $\beta$ subunits of brain spectrin among the total hrain membrane proteins, it could be used as a reliable probe for the immunocytochemical localization of brain spectrin within neural tissue.

\section{Immunofluorescent localization of spectrin antigens in brain}

Examination of sagittal and coronal sections of mouse brain stained with anti-RBC spectrin IgG revealed a specific pattern of immunoreactivity. Spectrin antigens were concentrated in the cell bodies of neurons and in cell processes. Synaptic structures and axons were observed to have little detectable spectrin analogues by immunofluorescence methodology. Glia 


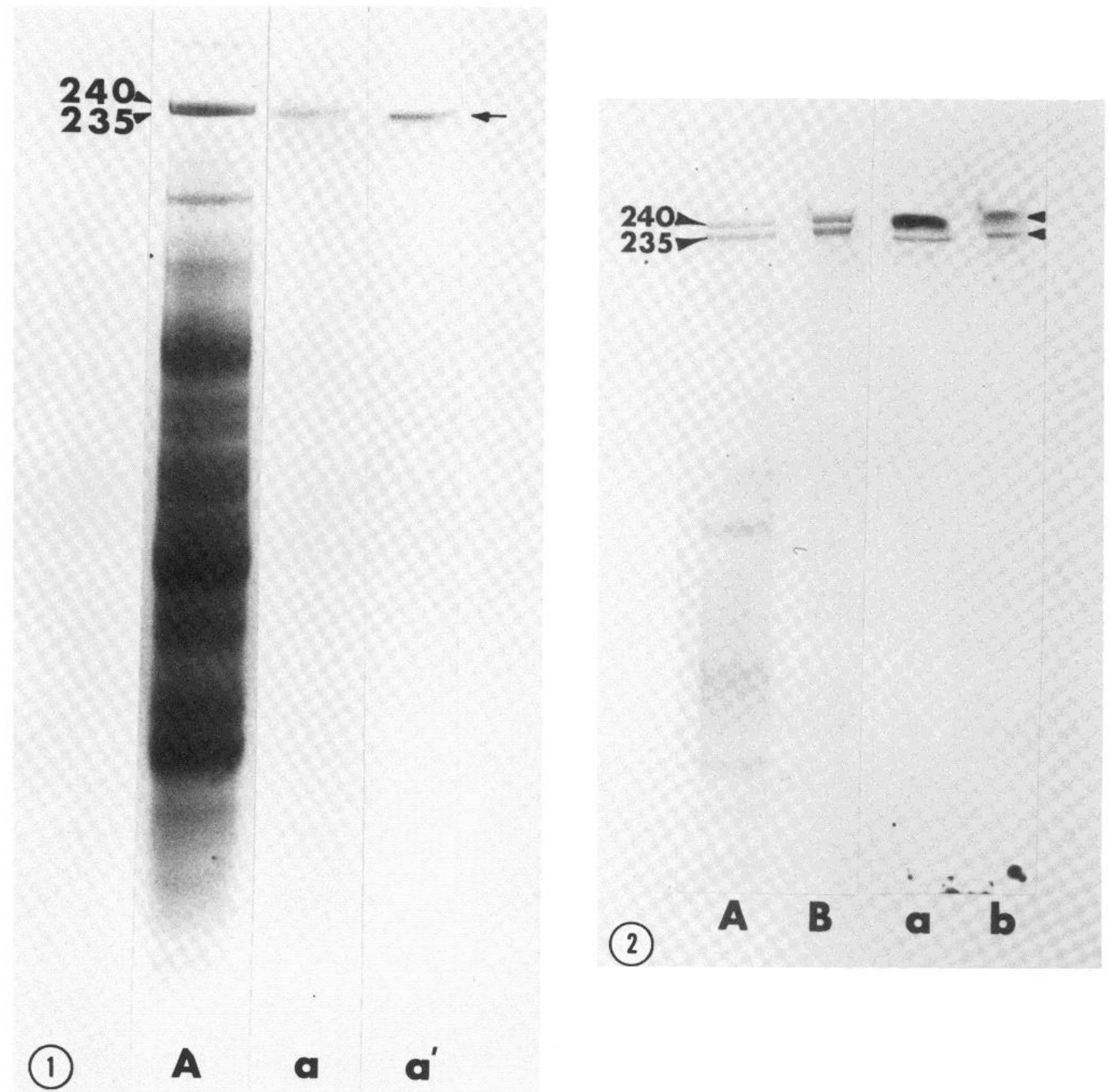

Figures 1 and 2. Immunoautoradiographic characterization of the anti-mouse RBC spectrin IgG.

Figure 1. SDS-PAGE and immunoautoradiography were performed as described under "Materials and Methods." Coomassie blue-stained demyelinated mouse brain membrane protein (lane $A$ ) was stained with anti-mouse $\mathrm{RBC}$ spectrin IgG followed by ${ }^{125} \mathrm{I}-\mathrm{Protein} \mathrm{A}$, and autoradiographs were exposed for 4 days $(a)$ or 8 days $\left(a^{\prime}\right)$ at $-20^{\circ} \mathrm{C}$. The brain spectrin $\alpha$ and $\beta$ subunits are designated 240 and 235 (molecular weight $\left.\times 10^{-3}\right)$. Specific staining of brain spectrin is designated with an arrow.

Figure 2. To ascertain whether the $M_{\mathrm{r}}=240,000$ or 235,000 subunits of mouse brain spectrin were stained, electrophoresis was extended for an additional $4 \mathrm{hr}$. The Coomassie blue-stained gel containing $(A)$ demyelinated brain membrane protein and $(B)$ purified mouse brain spectrin were stained with anti-RBC spectrin IgG and ${ }^{125} \mathrm{I}$-Protein A. The clearly separated 240,000 - and 235,000 -dalton subunits are designated by 240 and 235 on the gel and by arrowheads on the immunoautoradiograph. The autoradiographs $(a$ and $b)$ were exposed for 14 days at $-20^{\circ} \mathrm{C}$.

had a less intense immunoreactivity in their cell bodies, whereas glial processes were unstained. Cell nuclei of neural cells were not fluorescent. Of course, the immunofluorescence technique can only supply a qualitative view of relative spectrin content in different neural cells. For example, the less intense staining of neuronal axons or glial cell types could be due to a spectrin molecule that has less antigenic sites which can be recognized by our RBC spectrin IgG than the spectrin molecule found in the neuronal cell bodies. Given these general results, we have selected examples from our extensive investigation that formulate a more detailed account of our findings.

Cerebellum. Study of the cerebellar cortex stained with anti$\mathrm{RBC}$ spectrin IgG at low magnification revealed that the molecular layer (Figs. 3, 4, 6, 8, and 11) exhibited a low to moderate staining, but the Purkinje cell layer (Figs. 3 and 6 to 8 ) and the internal granule layer (Figs. 3,5 to 9 , and 11) were intensely fluorescent. Little immunoreactivity was recorded in the medullary layer (Figs. 3, 9, and 11). All neural cell types in the 

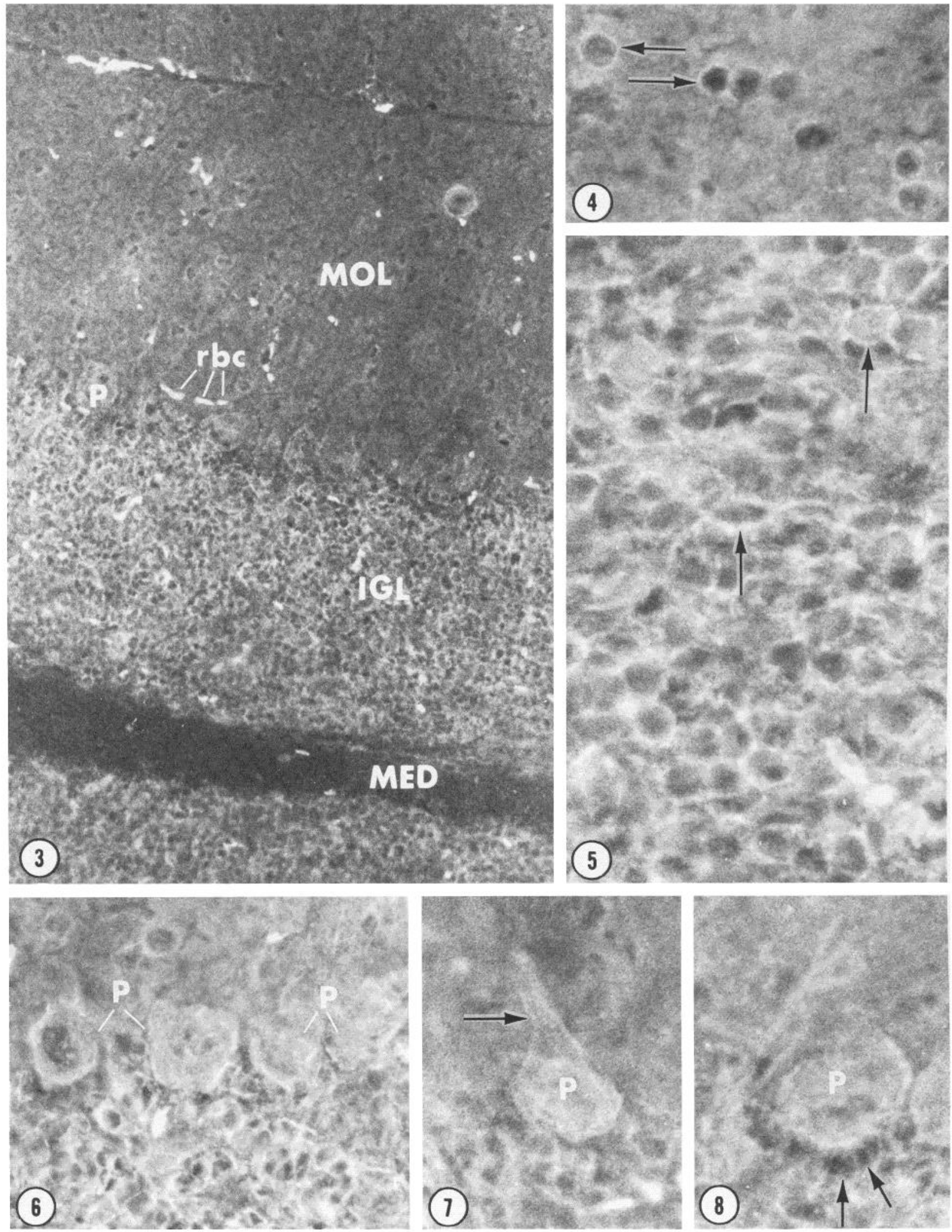

Figures 3 to 30 . All figures are of an adult mouse brain. Unless otherwise noted, all photomicrographs were obtained with fluorescent optics of tissues stained with anti-RBC spectrin IgG and rhodamine-conjugated goat anti-rabbit IgG.

Figures 3 to 15. Photomicrographs of sagittal sections of the cerebellar cortex.

Figure 3. A low magnification photomicrograph showing intense fluorescence in the internal granule layer $(I G L)$ and of the Purkinje cell $(P)$ and erythrocytes $(r b c)$. The molecular layer $(M O L)$ exhibited a low to moderate fluorescence whereas the medullary layer $(M E D)$ displayed little immunoreactivity. Magnification $\times 240$.

Figure 4. Neural cells in the molecular layer. In each case, note a bright fluorescence (arrows) bordering an unstained nucleus. Magnification $\times 650$. 

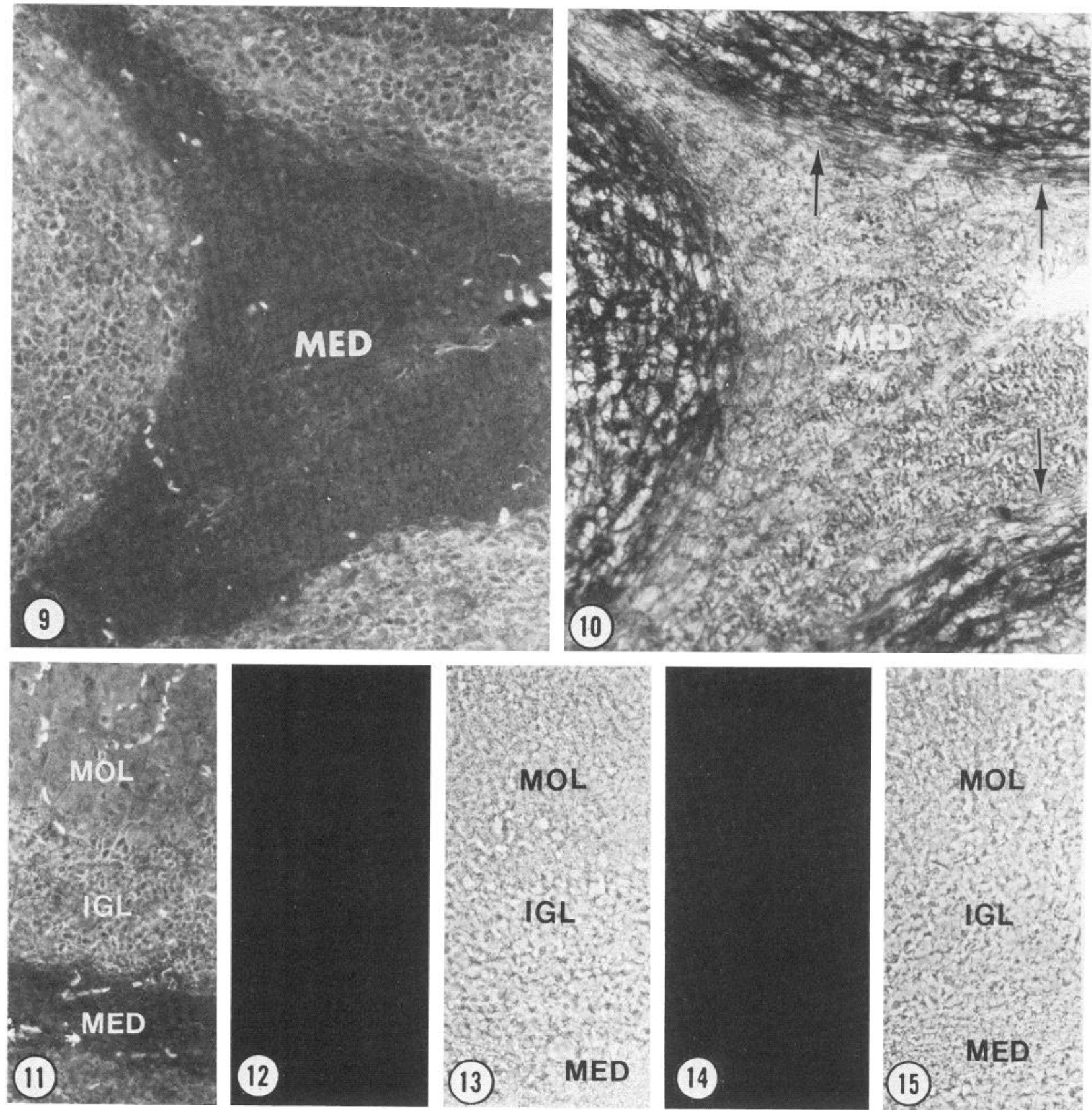

Figure 9. The medullary layer $(M E D)$ has only a slight fluorescence in comparison to the intense fluorescence in the three groupings of the internal granule layer bordering the medullary layer. Magnification $\times 260$.

Figure 10. Following examination of fluorescence staining recorded in Figure 9, this section was stained with a modification of the Protargol technique which is selective (Zagon and Haring, 1981) for neuronal elements. Note the argyrophilic fibers (arrows) coursing through the medullary layer (MED) and extending into the internal granule layer as a complex pattern of processes which are absent in the fluorescent images stained with anti-spectrin IgG. Magnification $\times 260$.

Figure 11 to 15. Photomicrographs of control specimens utilized for the specificity of spectrin immunofluorescence. Figure 11 is a low magnification photomicrograph of the cerebellar cortex stained with anti-RBC spectrin IgG to be used as a control for exposure and printing in specimens stained with preimmune IgG (Fig. 12) or brain spectrin absorbed anti-RBC spectrin IgG (Fig. 14); phase-contrast photomicrographs (Figs. 13 and 15 ) identify the areas examined in Figures 12 and 14, respectively. IGL, internal granule layer; $M E D$, medullary layer; $M O L$, molecular layer. Magnification $\times 175$.

Figure 5. Granule neurons in the internal granule layer. An intensely stained cortical cytoplasm (arrows) surrounds nuclei with little fluorescence. Magnification $\times 1050$.

Figure 6. Purkinje neurons $(P)$ exhibited prominent fluorescence of cortical cytoplasm, but their cell nuclei (see Purkinje cell on far left) appeared fluorescence negative. As recorded in Figure 3, the molecular layer located above the Purkinje neurons was of low to moderate intensity, whereas the granule cells (located beneath the Purkinje cells) exhibited a positive immunoreactivity in their cortical cytoplasm. Magnification $\times 540$.

Figure 7. The dendrite (arrow) of a Purkinje neuron $(P)$ and the cell body are stained with anti-RBC spectrin IgG. Magnification $\times 770$.

Figure 8 . A Purkinje cell $(P)$ partly encircled by elements that appear to be Bergmann glial cells (arrows). The nuclei of these glial cells were unstained, whereas a rim of fluorescence (presumably the cortical cytoplasm) could sometimes be recorded. Magnification $\times 800$. 

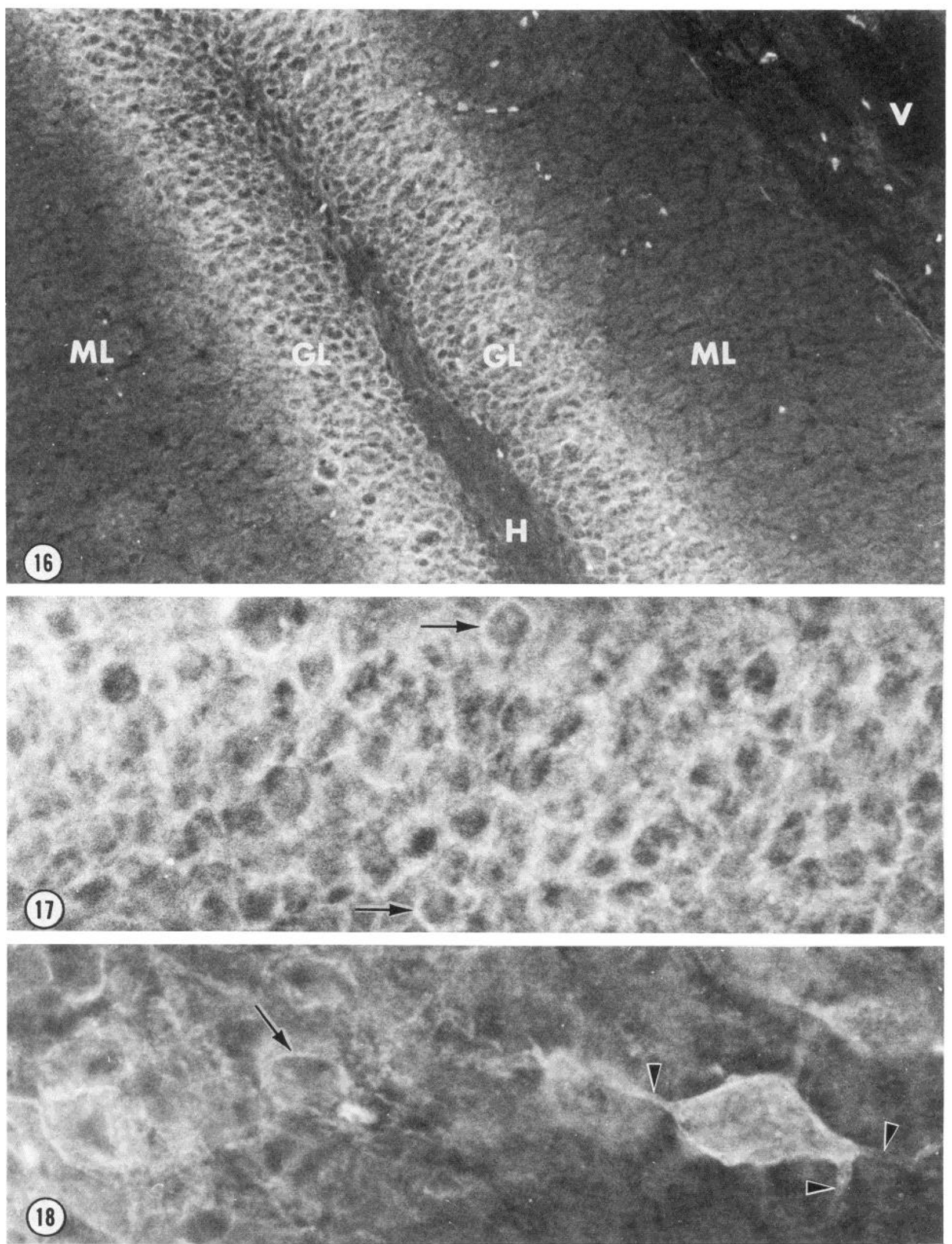

Figure 16. A low magnification photomicrograph of a sagittal section of the dentate gyrus. Cells in the granule layer $(G L)$ are extremely immunoreactive, whereas the hilus $(H)$ and molecular layer $(M)$ exhibit only slight fluorescence. $V$, ventricle. Magnification $\times 375$.

Figure 17. The granular layer of the dentate gyrus, recorded from a sagittal section of the brain, contains granule neurons with a bright cortical cytoplasm (arrows) but unstained nuclei. Magnification $\times 800$.

Figure 18. In this sagittal section of the hippocampal formation, an intensely stained cell with at least three major branches (arrowheads) can be observed. Note the bright cytoplasm (arrow) encircling a dark nucleus of a pyramidal neuron. Magnification $\times 900$. 

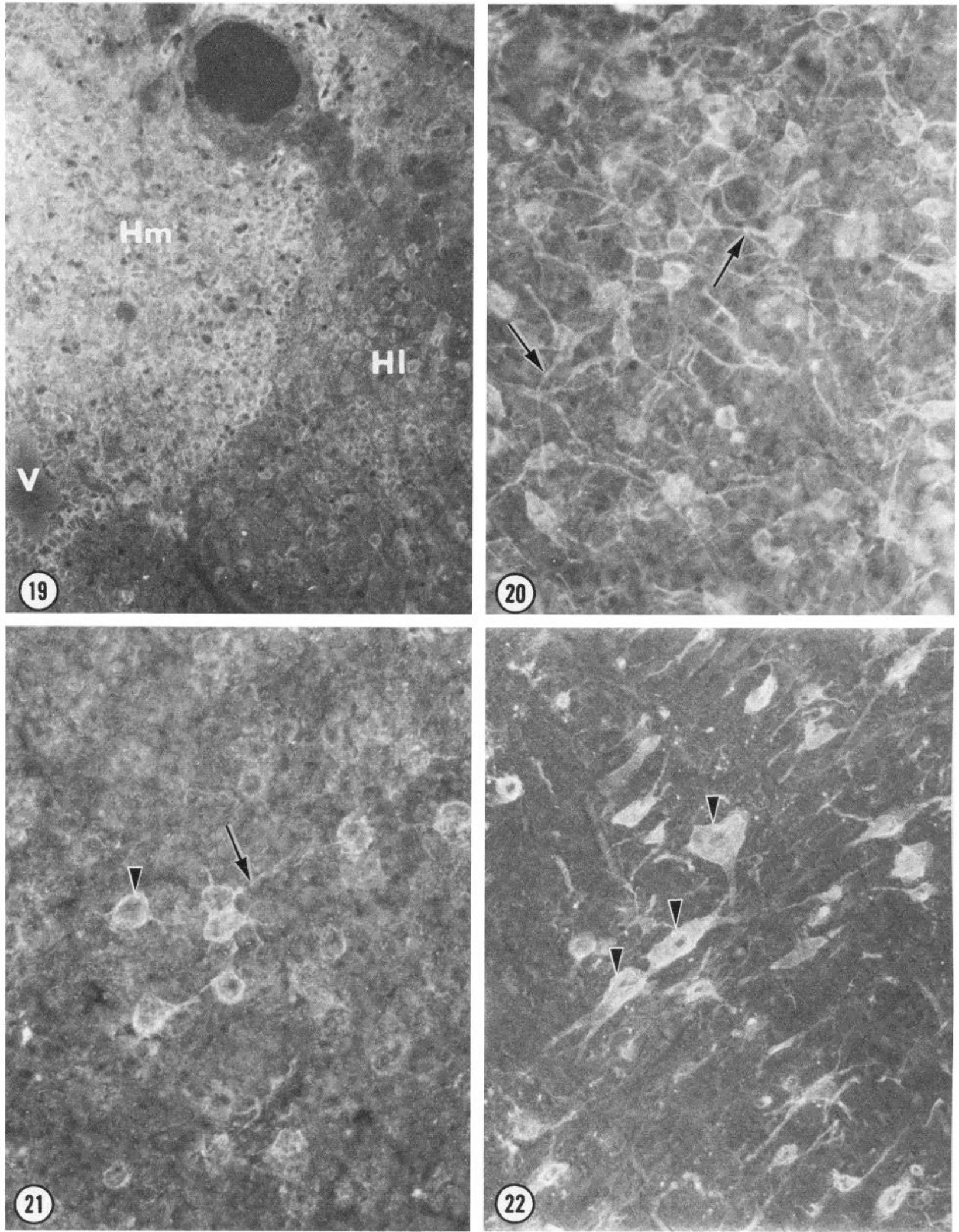

Figure 19. The medial habenula $(\mathrm{Hm})$ contains numerous immunoreactive neural cells. In contrast, the lateral habenula $(\mathrm{Hl})$, an area more sparsely populated with neural cell bodies, was of lower immunoreactivity. Coronal section. Magnification $\times 165$.

Figure 20. Fluorescent neural cells, presumably neurons, with long processes (arrows) in the thalamus. Coronal section. Magnification $\times 260$.

Figure 21. Neural cells in the cerebral cortex exhibit a prominent fluorescence of the cortical cytoplasm (arrowhead); nuclei were unstained. Extremely fine fluorescent processes (arrow) can be recorded. Coronal section. Magnification $\times 410$.

Figure 22. Brightly fluorescent cellular elements (arrowheads) in the brainstem. With the exception of the nucleus, cell bodies and processes were stained with anti-spectrin. Sagittal section. Magnification $\times 260$. 

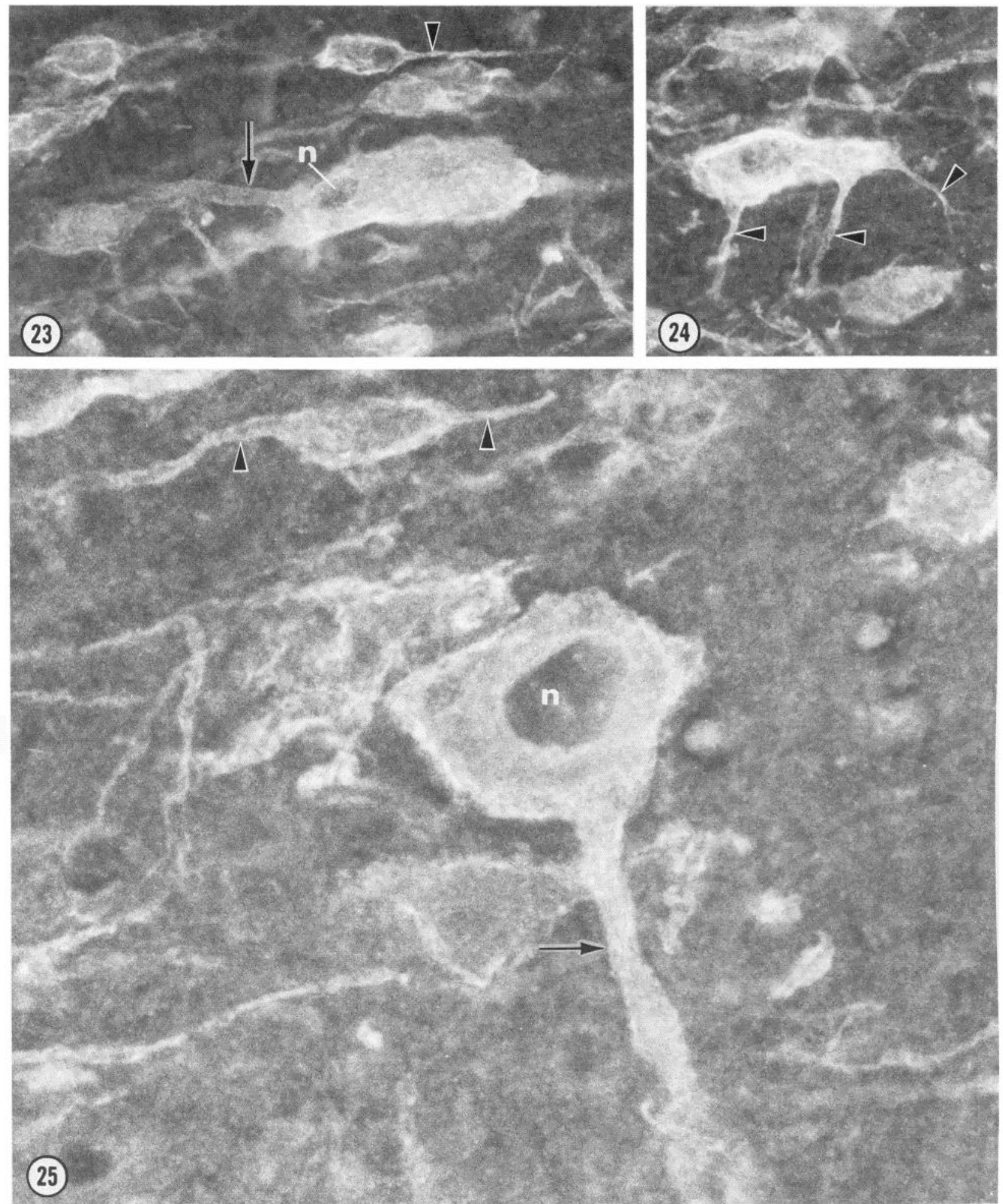

Figures 23 to 25 . High magnification photomicrographs of neural elements in the brainstem recorded in sagittal sections to show the details of neural cell immunoreactivity.

Figure 23. A large, fluorescent neural cell with a dark nucleus $(n)$ has a number of processes (arrow) emanating from the soma. Note the extremely small process (arrowhead) of an adjacent cell. Magnification $\times 525$.

Figure 24. An intensely staining neural cell with numerous fine branches (arrowheads). Magnification $\times 525$.

Figure 25. A large neuron with bright fluorescence in the cell body and the cell process (arrow). The nucleus $(n)$ is relatively unstained. Note that the staining pattern in the cell process appears to be organized as discrete strands of fluorescence located parallel to the long axis of the process. An adjacent bipolar neural cell has immunoreactive processes (arrowheads) emanating from its fluorescence-positive soma. Magnification $\times 1050$. 

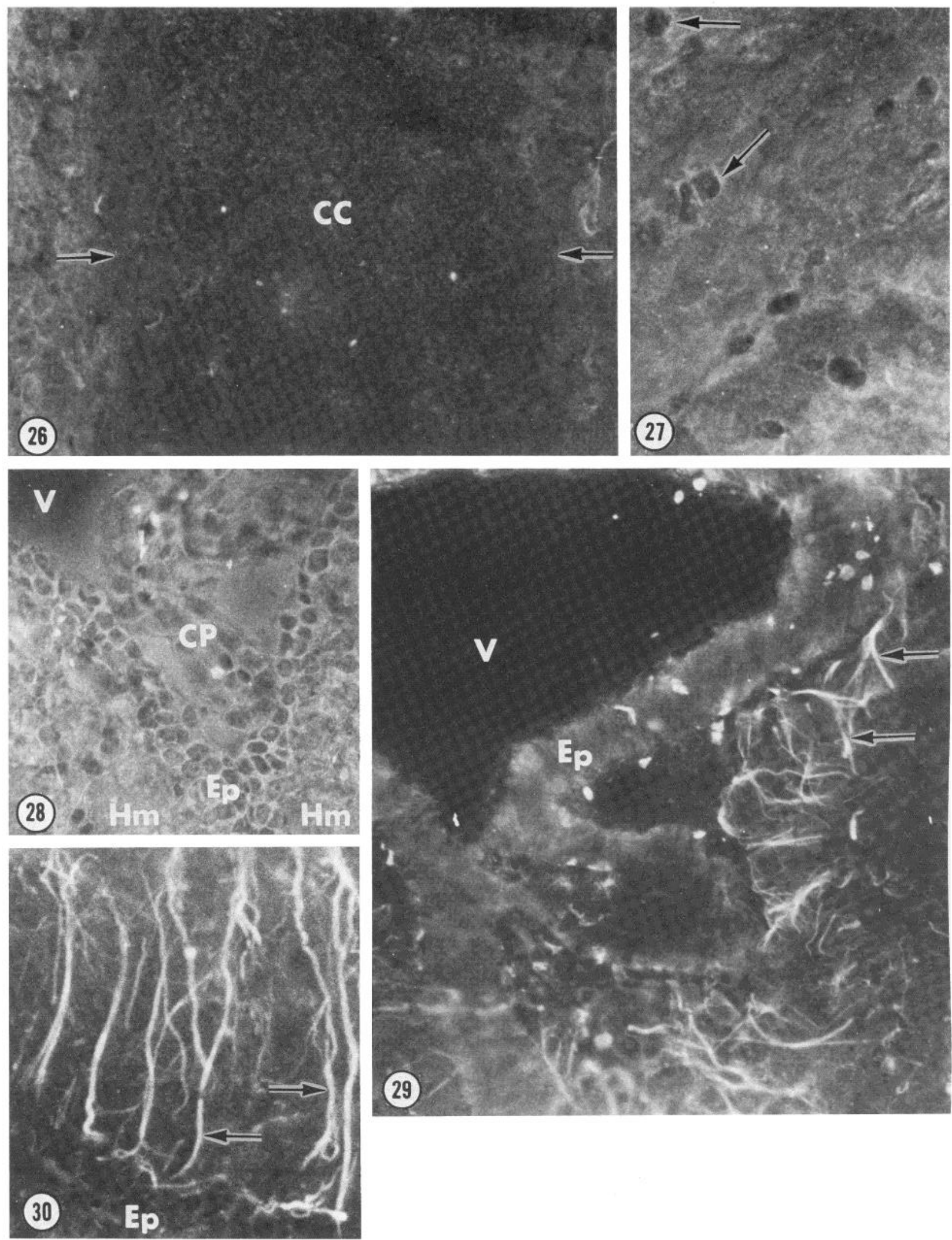

Figure 26. Sagittal section of the corpus callosum ( $c c$; boundaries indicated by the arrows) illustrating slight fluorescence staining. Magnification $\times 400$.

Figure 27. Glial cells in a coronal section of the corpus callosum. The cells exhibit a rim of fluorescence (arrows) that encircles an unstained nucleus. Magnification $\times 580$.

Figure 28. Cells of the choroid plexus $(C P)$ can be observed extending into the third ventricle $(V)$. The cortical cytoplasm of ependymal cells $(E p)$ is extremely immunoreactive. Part of the medial habenula $(\mathrm{Hm})$ (see also Fig. 19) can be observed. Coronal section. Magnification $\times 300$.

Figure 29. Brightly fluorescent fibers (arrows) were often recorded subjacent to the ependyma $(E p)$. $V$, lateral ventricle. Sagittal section. Magnification $\times 330$.

Figure 30. Long, intensely fluorescent strands (arrows) located subjacent to the ependyma (Ep). Lateral ventricle, sagittal section. Magnification $\times 560$. 
molecular layer had a distinct rim of fluorescence surrounding an unstained nucleus (Fig. 4). The neuropil of the molecular layer was only of low to moderate immunoreactivity (Figs. 3, 4, and 6 to 8 ). The cytoplasm of internal granule neurons was intensely fluorescent, but granule cell nuclei were fluorescence negative (Figs. 5, 6, 9, and 11). The soma of Purkinje neurons also had a bright fluorescence (Figs. 6 to 8 ). In sections passing through the plane of the nucleus, this structure had a notable lack of staining (Fig. 6). The trunk of the Purkinje cell dendrite was clearly stained with anti-RBC spectrin (Fig. 7), but smaller dendritic branches were not recorded. Axons of Purkinje neurons were below the level of detection. In some sections, a distinct group of small, dark, circular profiles occasionally containing a lightly stained perinuclear cytoplasm was recorded (Fig. 8); these cells were tentatively identified as Bergmann glia.

In contrast to other regions of the cerebellar cortex, the medullary layer demonstrated extremely weak fluorescence (Figs. 3, 9, and 11). Following examination of the medullary layer with fluorescence optics, sections were processed for Protargol staining (Fig. 10). In contrast to fluorescence images, numerous argyrophilic fibers (i.e., axons of Purkinje neurons and climbing and mossy fibers) were observed in the medullary layer, and these nerve fibers formed a complex network in the internal granule layer that extended up to the Purkinje layer. Occasionally, some fluorescent material, often organized as strands or tubular structures, was visible in the medullary layer; this material did not correspond to axons as evaluated in Protargol-stained specimens.

A series of control sections was included for our studies (Figs. 11 to 15). The staining patterns were specific for spectrin in the cerebellum, as well as the other brain regions. Sections stained with preimmune IgG (Fig. 12) or anti-RBC spectrin preabsorbed with a 10 -fold excess of RBC spectrin (Fig. 14) exhibited little or no staining.

Hippocampal formation. Low magnification photomicrographs of the dentate gyrus (Fig. 16) revealed a staining profile similar to that of the cerebellum. The granular layer demonstrated a prominent fluorescence in contrast to the low level of staining in the molecular layer and hilar regions. Close inspection of the granule cells showed that the cytoplasm was stained with anti-spectrin IgG, with little detectable immunofluorescence in cell nuclei (Fig. 17). Pyramidal cells were stained in a manner resembling that of granule neurons (Fig. 18). In addition, a number of adjacent, nonpyramidal cell types which were tentatively identified as neurons had a prominent immunofluorescence (Fig. 18).

Other brain regions. In addition to the cerebellum and hippocampal formation, comprehensive examination of sagittal and coronal sections showed that neural cells in all brain regions appeared to contain spectrin (Figs. 19 to 25). For example, the medial habenula, which has a dense packing of neural cells, displayed intense staining. In contrast, the lateral habenula, which was sparsely populated with cells, had a low level of fluorescence. Our studies showed that cell bodies and cell processes were stained with anti-spectrin IgG (Figs. 19 to 25) but the surrounding neuropil had little immunoreaclivity, and cell nuclei were fluorescence negative. High magnification inspection of neural tissues (Figs. 23 to 25) confirmed these observations and revealed that staining extended into the finest neuronal processes. However, staining of synaptic boutons was not evident. In a number of instances, particularly in large cell processes, the staining pattern appeared to be organized into strand-like arrays (Fig. 25). Neural cell staining usually appeared to be very dense in regions associated with the plasma membrane and was especially notable when the cell was within the plane of section (Figs. 4 to $6,17,19$ to 22, and 25). However, staining of the "cytoplasm" was evident in both "sectioned" and "intact" cells (Figs. 6 to 8, 18, 20, and 22 to 25). Because of the limits of resolution with light microscopy, we could not determine whether all of the "cytoplasmic" staining was submembranous.

Glia and myelin tracts. As noted earlier, especially in our inspection of the cerebellar medullary layer, areas with myelin were only slightly stained with anti-RBC spectrin IgG. Examination of other regions with myelinated tracts confirmed this finding. For example, the corpus callosum (Fig. 26) exhibited little fluorescence, as did other regions including the mammillothalamic tract, fornix, internal capsule, corticospinal tract, anterior commissure, and cerebellar peduncles. Examination of the caudate putamen showed grouping of bright fluorescence interspersed with areas of equal size that were unstained. Correlation with Protargol and gallocyanin preparations revealed that fluorescence-stained area contained neurons whereas the unstained areas contained fascicles of internal capsule axons.

$\Lambda$ s mentioned earlier, the cytoplasm adjacent to the cell nuclei of glial cells was moderately fluorescent, but neither cell nuclei nor glial processes were stained with anti-RBC spectrin IgG. Photomicrographs (Figs. 26 and 27) of the corpus callosum serve to illustrate this observation.

Choroid plexus and ependyma. The choroid plexus (Fig. 28) had low to moderate immunoreactivity, but the cortical cytoplasm of ependymal cells was distinctly fluorescent. In the ventricular region, prominently stained fibrous elements were often observed (Figs. 29 and 30 ). These fibers were usually observed to extend from a region subjacent to the ependyma out a short distance into the surrounding brain tissue. The origin of these fibers was indeterminate.

\section{Discussion}

The complete study of spectrin localization in mammalian brain which is described above allows us to derive certain basic principles: (1) spectrin is found in all regions of mammalian brain and its intensity corresponds to the density of neural cells; (2) different neural cell types contain variable spectrin content-for example, neurons contain more immunoreactive spectrin than do glial cell types; (3) within a single cell type, the regional disposition of spectrin varies-for example, neurons display a brightly stained cytoplasm of the cell body and dendrites, little staining of axons, and no nuclear staining. To obtain a specific intercellular localization for spectrin in neural cells, we are currently performing electron microscopy of mouse brain samples stained with ferritin-labeled anti-RBC and brain spectrin IgG.

Willard and colleagues have described a protein which is transported down retinal ganglion axons at a velocity of 40 $\mathrm{mm} /$ day and contains subunits of 240,000 and 235,000 daltons (Lorenz and Willard, 1978). Antibodies raised against this protein, termed fodrin, stained the periphery of neuronal cell bodies, dendrites, and axons in the peripheral nervous system (Levine and Willard, 1981). It has been suggested that fodrin may be a component of a mobile axonal lining which is in continuous motion away from the cell body and which is essential to the axonal transport of various organelles (Cheney et al., 1983). Since work by others has demonstrated that fodrin is immunologically and structurally related to $\mathrm{RBC}$ spectrin (Bennett et al., 1982; Burridge et al., 1982; Glenney et al., 1982b; Goodman et al.,1983, 1984a; Casoria et al., 1984), most investigators in this field currently refer to this spectrin-like protein as brain spectrin. Based on Willard's elegant studies, we anticipated seeing bright staining of the neuronal cell body, dendrites, and axons in mouse brain tissue, but, surprisingly, we found little staining of elements which could be unequivocally identified as axons in the central nervous system. However, Willard's immunofluorescence studies were performed on 
guinea pig peripheral nervous system, utilizing an antibody against brain spectrin (fodrin) which stained the $\alpha$ subunit of brain spectrin. Our immunofluorescence studies were performed in mouse brain central nervous system, utilizing a monospecific antibody against mouse RBC spectrin which stained both the 240,000- and 235,000-dalton $\alpha$ and $\beta$ subunits of brain spectrin. Therefore, differences in axonal staining could potentially be due to species differences, antibody specificity, or differences in the content of spectrin in axons from the peripheral and central nervous systems. We have performed indirect immunofluorescence studies on sciatic nerve utilizing antibodies against mouse RBC spectrin and mouse brain spectrin (data not shown), and the images of axonal staining in both cases were identical to those presented by Levine and Willard (1981). We therefore feel that the low level of axonal staining observed in the mouse central nervous system with spectrin antibodies is not due to species or antibody specificity but instead is due to real differences in the spectrin content of axons and/or other associated elements (e.g., Schwan cells, oligodendrocytes) of the central and peripheral nervous systems. What can be clearly stated is that in mouse brain, the content of spectrin antigens in neuronal cell bodies and dendrites is greater than the spectrin content of axons.

Two brief reports on chicken brain spectrin have recently appeared which suggest that in avian brain the major form of brain spectrin (fodrin, 240,000 to 235,000 daltons) is present in all neuronal cell bodies and processes and that this form of brain spectrin is expressed during all stages of cerebellar morphogenesis (Lazarides and Nelson, 1983a, b). In addition, these authors have suggested that a minor form of chicken brain spectrin, which has a 230,000 -dalton $\beta^{\prime}$ subunit closely related to $\mathrm{RBC} \beta$ spectrin (associated with the $\alpha$ subunit), is confined to the plasmalemma of neuronal cell bodies and is expressed only in postmitotic cells in response to formation of functional synaptic connections. Several points need to be made. First, the history of the nonerythroid spectrin field has demonstrated that one cannot easily extrapolate information obtained in avian systems to mammalian systems. For example, whereas all mammalian RBC spectrins contain a 240,000-dalton $\alpha$ subunit in association with a 220,000 -dalton $\beta$ subunit (Whitfield et al., 1984; for review, see Goodman and Shiffer, 1983), chicken RBC spectrin contains a 220,000-dalton $\beta$ subunit and a 230,000 -dalton $\beta^{\prime}$ subunit individually associated with a 240,000-dalton $\alpha$ subunit (Lazarides and Nelson, 1983a). In addition, whereas all nonerythroid spectrins from chicken contain an $\alpha$ subunit which is identical by peptide mapping analysis and a variable $\beta$ subunit, the mammalian spectrins demonstrate variable $\alpha$ and $\beta$ subunits (see Goodman and Shiffer, 1983, and Glenney and Glenney, 1983a, for reviews). Thus far, no evidence for a minor form of spectrin containing a 230,000-dalton $\beta^{\prime}$ subunit in association with an $\alpha$ subunit has been demonstrated in mammalian brain. Second, our spectrin antibody, which cross-reacts with both the 240,000 -dalton $\alpha$ subunit and the 235,000 -dalton $\beta$ subunit of mouse brain spectrin, would detect both the major $240,000 / 235,000$-dalton brain spectrin (fodrin) and any minor 240,000/230,000-dalton spectrin component, if indeed the minor component existed in mouse brain. Therefore, the immunofluorescent images presented in this paper are displaying the localization of "total" brain spectrin. Currently, we are testing for the presence of the minor brain spectrin molecules in mouse utilizing antibodies which are raised against mouse $\mathrm{RBC} \alpha$ subunit or mouse $\mathrm{RBC} \beta$ subunit in immunoprecipitation, immunoautoradiography, and immunofluorescence studies. In conclusion, to understand the function and disposition of spectrin in mammalian brain, one must study mammalian brain, rather than extrapolating from avian systems.

Although brain is a nonerythroid tissue which is rich in spectrin, the content of spectrin is quite variable in distinct neural cells. If spectrin's function in brain is similar to its function in the highly specialized erythrocyte, then we would expect the spectrin in neurons to control cell shape and elasticity, membrane structural integrity, lateral mobility of membrane proteins and lipids, and transbilayer movement of molecules, and to link actin to the plasma membrane. Recently, a protein 4.1 analogue has been demonstrated to co-localize with spectrin in mammalian brain (Goodman et al., 1984b); this brain protein 4.1 may be essential to the brain spectrin-actin interaction. In the future, we need to obtain a higher resolution view of spectrin in brain by immunoelectron microscopy, and we need to isolate and compare neuronal and glial spectrins.

\section{References}

Bennett, V., and P. J. Stenbuck (1979) Identification and partial purification of ankyrin, the high affinity membrane attachment site for human erythrocyte spectrin. J. Biol. Chem. 254: 2533-2541.

Bennett, V., and P. J. Stenbuck (1980) Human erythrocyte ankyrin: Purification and properties. J. Biol. Chem. 255: 6424-6432.

Bennett, V., J. Davis, and W. E. Fowler (1982) Brain spectrin, a membrane-associated protein related in structure and formation to erythrocyte spectrin. Nature 299: 126-131.

Burns, N. R., V. Ohanian, and W. B. Grater (1983) Properties of brain spectrin (fodrin). FEBS Lett. 153: 165-168.

Burridge, K., T. Kelly, and R. Mangeat (1982) Nonerythrocyte spectrins: Actin-membrane attachment proteins occurring in many cell types. J. Cell Biol. 95: 478-486.

Carlin, R. K., D. C. Bartelt, and P. Siekevitz (1982) Identification of fodrin as a major calmodulin-binding protein in postsynaptic density preparations. J. Cell Biol. 95: 478-486.

Casoria, L. A., I. S. Zagon, S. E. Bernstein, S. B. Shohet, P. J. McI aughlin, and S. R. Goodman (1984) Normal content of hrain spectrin-like protein in sph/sph mice. Br. J. Hematol., in press.

Cheney, R., N. Hirokawa, J. Levine, and M. Willard (1983) Intracellular movement of fodrin. Cell Motil. 3: 649655.

Einarson, L. (1951) On the theory of gallocyanin-chromalum staining and its application for quantitative estimation of basophilia. A selective staining of exquisite progressivity. Acta Pathol. Microbiol. Scand. 28: 82-102.

Fairbanks, G., T. L. Steck, and D. F. H. Wallach (1971) Electrophoretic analyses of the major polypeptides of the human erythrocyte membrane. Biochemistry 10: 2606-2617.

Glenney, J. R., Jr., and P. Glenney (1983a) Spectrin, fodrin, and TW 260/240: A family of related proteins lining the plasma membrane. Cell Motil. 3: 671-682.

Glenney, J. R., Jr., and P. Glenney (1983b) Fodrin is the general spectrin-like protein found in most cells whereas spectrin and the TW Protein have a restricted distribution. Cell 34: 503-512.

Glenney, J. R., Jr., P. Glenney, M. Osborn, and K. Weber (1982a) An F-actin- and calmodulin-binding protein from isolated intestinal brush borders has a morphology related to spectrin. Cell 28: 843854 .

Glenney, J. R., Jr., P. Glenney, and K. Weber (1982b) Erythroid spectrin, brain fodrin, and intestinal brush border proteins (TW$260 / 240$ ) are related molecules containing a common calmodulinbinding subunit bound to a variant cell type-specific subunit. Proc Natl. Acad. Sci. U. S. A. 79: 4002-4005.

Glenney, J. R., Jr., P. Glenney, and K. Weber (1982c) F-actin binding and cross-linking properties of porcine brain fodrin, a spectrinrelated molecule. J. Biol. Chem. 257: 9781-9787.

Goodman, S. R., and K. Shiffer (1983) The spectrin membrane skeleton of normal and abnormal erythrocytes: a review. Am. J. Physiol. 244 C121-C141.

Goodman, S. R., I. S. Zagon, and R. R. Kulikowski (1981) Identification of a spectrin-like protein in nonerythroid cells. Proc. Natl. Acad. Sci. U.S. A. 78: $7570-7574$.

Goodman, S. R., I. S. Zagon, C. F. Whitfield, L. A. Casoria, P. J. McLaughlin, and T. L. Laskiewicz (1983) A spectrin-like protein from mouse brain membranes: Immunological and structural correlations with erythrocyte spectrin. Cell Motil. 3: 635-647.

Goodman, S. R., I. S. Zagon, C. F. Whitfield, L. A. Casoria, S. B. Shohet, S. E. Bernstein, P. J. McLaughlin, and T. L. Laskiewicz (1984a) A spectrin-like protein from mouse brain membranes: Phos- 
phorylation of the 235,000 dalton subunit. Am. J. Physiol. 247: C61C73.

Goodman, S. R., L.A. Casoria, D. B. Coleman, and I. S. Zagon (1984b) Identification and localization of brain protein 4.1. Science 224 : $1433-1436$.

Kakiuchi, S., K. Sobue, and M. Fujita (1981) Purification of a 240,000 $M_{\mathrm{r}}$ calmodulin-binding protein from a microsmal fraction of brain. FEBS Lett. 132: 144-148.

Kakiuchi, S., K. Sobue, K. Morimoto, and K. Kanda (1982) A spectrinlike calmodulin-binding protein (Calspectin) of brain. Biochem. Int. 5: $755-762$.

Lazarides, E., and W. J. Nelson (1983a) Erythrocyte and brain forms of spectrin in cerebellum: Distinct membrane-cytoskeletal domains in neurons. Science 220; 1295-1296.

Lazarides, E., and W. J. Nelson (1983b) Erythrocyte form of spectrin in cerebellum: Appearance at a specific stage in the terminal differentiation of neurons. Science 222: 931-933.

Levine, J., and M. Willard (1981) Fodrin: Axonally transported polypeptides associated with the internal periphery of many cells. J. Cell Biol. 90: 631-643

Lorenz, T., and M. Willard (1978) Subcellular fractionation of intraaxonally transported polypeptides in the rabbit visual system. Proc. Natl. Acad. Sci. U. S. A. 75: 505-509.

Palfrey, H. C., W. Schiebler, and P. Greengard (1982) A major calmodulin-binding protein common to various vertebrate tissues. Proc. Natl. Acad. Sci. U. S. A. 79: 3780-3784.

Repasky, E. A., B. L. Granger, and E. Lazarides (1982) Widespread occurrence of avian spectrin in nonerythroid cells. Cell 29: 821-833.

Shiffer, K. A., and S. R. Goodman (1983) The association between human erythrocyte protein 4.1 and the erythrocyte membrane. Fed. Proc. 42: 2700.

Shiffer, K. A., and S. R. Goodman (1984) Protein 4.1: Its association with the human erythracyte membrane. Proc. Natl. Acad. Sci. U. S. A. 81 : $4404-4408$.

Shimo-Oka, T., and Y. Watanbe (1981) Stimulation of actomyosin $\mathrm{Mg}^{2+}$-ATPase activity by a brain microtubule-associated protein fraction. J. Biochem. 90:1297-1307.

Shotton, D. M., B. E. Burke, and D. Branton (1979) Molecular structure of human erythrocyte spectrin: Biophysical and elcctron microscopic studies. J. Mol. Biol. 131: 303-329.

Steck, T. L. (1974) The organization of proteins in the human red blood cell membrane. J. Cell Biol. 62: 1-19.

Whitfield, C. F., D. B. Coleman, M. M. B. Kay, K. Shiffer, J. Miller, and S. R. Goodman (1984) Characterization and interrelationships of the polypeptides of human red cell membrane zone 4.5. In Erythrocyte Membranes 3: Recent Clinical and Experimental Advances, W. C. Kruckenberg, J. W. Eaton, and G. J. Brewer, eds., Alan R. Liss, Inc., New York, in press.

Yu, J., and S. R. Goodman (1979) Syndeins: The spectrin binding protein(s) of the human erythrocyte membrane. Proc. Natl. Acad. Sci. U. S. A. 76: 2340-2344.

Zagon, I. S., and J. H. Haring (1982) Protargol silver impregnation and neuronal selectivity. Light and electron microscopic observations. Acta Anat. 114: 193-210.

Zagon, I. S., and R. S. Lasher (1977) A modification of the Bodian technique for embedded, frozen, and cultured nervous tissue. Trans. Am. Microsc. Soc. 96: 91-96.

Zagon, I. S., and P. J. McLaughlin (1981) Naloxone prolongs the survival time of mice treated with neuroblastoma. Life Sci. 28: 10951102. 